\title{
Increasing the Precision of Hypertension Treatment Through Personalized Trials: a Pilot Study
}

\author{
Ian M. Kronish, $M D, M P H^{7}$, Ying Kuen Cheung, $P h D^{2}$, Daichi Shimbo, $M D^{7}$, \\ Jacob Julian, $B A^{7}$, Benjamin Gallagher, $M D^{3}$, Faith Parsons, $M S^{7}$, and \\ Karina W. Davidson, $P h D^{7}$
}

${ }^{1}$ Center for Behavioral Cardiovascular Health, Columbia University Irving Medical Center, New York, NY, USA; ${ }^{2}$ Department of Biostatistics, Mailman School of Public Health, Columbia University, New York, USA; ${ }^{3}$ Section of General Internal Medicine, Department of Internal Medicine, Yale School of Medicine, Yale University, New Haven, CT, USA.

BACKGROUND: There are substantial differences in the effects of blood pressure (BP) medications in individual patients. Yet, the current standard approach to prescribing BP medications is not personalized.

OBJECTIVE: To determine the feasibility of individualizing the selection of BP medications through pragmatic personalized (i.e., $N$-of-1) trials.

DESIGN: Series of $N$-of- 1 trials.

SETTING: Outpatient.

PATIENTS: Hypertensive adults prescribed none or one BP medication.

INTERVENTION: Participation in a flexible, open-label personalized trial of two to three BP medications (NCT02744456).

MEASUREMENTS: BP was measured twice per day with a validated home BP device. Frequency and severity of side effects were assessed at the end of the day via an electronic questionnaire. Patients' BP medication preference was assessed after reviewing BP lowering and side effect results with a study clinician. Feasibility was assessed by determining the proportion of patients who adhered to self-assessments. Benefit was assessed by asking patients to rate the helpfulness of participation and whether they would recommend personalized trials to other hypertensive patients.

KEY RESULTS: Of ten patients enrolled, two dropped out prior to initiation, one discovered white coat hypertension through ambulatory BP monitoring, and seven (mean age 58 years, $71 \%$ of women) completed personalized trials. All seven were compliant with home BP monitoring and side effect tracking. All seven recommended personalized trials of BP medications to others. Thiazides were preferred by three patients, renin-angiotensin system-blocking agents by two patients, a combination of thiazide and beta-blocker by one patient, and any of three classes by one patient.

CONCLUSIONS: Personalized trials of BP medications were feasible and led to improved treatment precision. Heterogeneity of patient preferences and of therapeutic $\mathrm{BP}$ response for first-line BP medications can be determined through a personalized trial approach.

Electronic supplementary material The online version of this article (https://doi.org/10.1007/s11606-019-04831-z) contains supplementary material, which is available to authorized users.

Received April 3, 2018

Revised September 6, 2018

Accepted December 5, 2018

Published online March 11, 2019

KEY WORDS: hypertension; primary care; clinical trials; disease management; health care delivery.

J Gen Intern Med 34(6):839-45

DOI: $10.1007 /$ s 11606-019-04831-Z

(c) Society of General Internal Medicine 2019

I $\mathrm{n}$ the traditional approach to treating hypertension, clinicians start a patient on a blood pressure (BP) medication from one of four guideline-recommended drug classes with little patient input into the choice of medication. ${ }^{1}$ Subsequent treatment decisions rely on BP readings from sporadic office visits and side effect assessments that are retrospective and thus prone to recall bias. Further, medications are usually added rather than switched, and nearly half of treated patients are prescribed several antihypertensive medications to get to goal. $^{2}$ Yet, multiple studies show that there are substantial differences in the way that antihypertensive medications affect BP in individual patients. ${ }^{3-6}$ Unfortunately, efforts to individualize the selection of BP medications through genetic or hormonal tests have not yet been successful. ${ }^{7,8}$ Thus, clinicians currently have no way of knowing which medications are best for individual patients without having the patients try the medications first, leading to the trial-of-therapy approach that is common in present-day practice.

Personalized trials (i.e., $N$-of-1 trials) may provide a more precise and scientific approach for selecting BP medications. Key features include a within-subjects crossover design, systematic collection of data on treatment effects with real-time objective measures, and visualization of data such that patients can share in decision-making about the relative benefits and harms of treatments. ${ }^{9,10}$ When conducted successfully, such trials provide the highest level of evidence for clinical decision-making. ${ }^{11,12}$

Thus, we tested the feasibility and perceived helpfulness of pragmatic personalized trials of BP medications that could be implemented in primary care. Our trials employed home BP monitoring to rigorously assess the effect of medications on BP, overcoming the limitations of relying solely on office 
measures. ${ }^{13}$ Our trials also used automated text messaging or emails to collect data on side effects conveniently and in real time, reducing recall bias. Finally, our trial allowed for customization based on patient preferences for a number of medications to compare and total study duration. Despite the intuitive appeal of this pragmatic approach, few have studied the feasibility of personalized trials of BP medications in a clinical context.

\section{METHODS}

\section{Personalized Trial Design}

The design of our personalized trial sought to balance rigor as specified by the CONSORT extension for reporting $\mathrm{N}$-of-1 trials (CENT) with patient centeredness and flexibility. ${ }^{14}$ The prototypical trial was designed to last 12 weeks and consisted of a counterbalanced sequence (i.e., ABCCBA) of three BP medications from three different first-line BP classes (hydrochlorothiazide (HCTZ), a thiazide diuretic; amlodipine, a calcium channel blocker; and losartan, a renin-angiotensin system-blocking agent), all prescribed as generic medications and each taken for 2 weeks at a time with one repetition each. A counterbalanced sequence was selected a priori to offset the risk of bias from time effects on BP. ${ }^{15}$ The design was adjusted after the study clinician met with the patient to incorporate patient preferences. If the patient was already taking a BP medication, the patient had the option of including that medication in the trial, and comparator medications were prescribed at equipotent doses. Also, patients had the option of comparing two or three medications, with the understanding that increasing the number of medications would increase the total duration of the trial. After trying each medication once (for 2 weeks at a time), patients had the option of reviewing their data and completing the trial without repetitions. Thus, pragmatic patient-centered features of the design included open-labeling, customized treatment selection, and adaptable treatment sequences.

\section{Setting and Patients}

We recruited patients by advertising the study on clinical trial websites (Clinicaltrials.gov and RecruitMe [url: https://recruit. cumc.columbia.edu]) and publicizing the study at primary care clinics affiliated with an academic medical center (Columbia University Irving Medical Center). Patients were eligible if they were 18 years to 80 years of age, if they had a history of mild hypertension (prescribed no BP medications and office BP greater than 140/90 $\mathrm{mmHg}$ or one BP medication and office BP $<180 / 110 \mathrm{mmHg}$ ), and if they had an established relationship with a medical provider who assented to patient participation in the study. Patients were excluded if their office BP was greater than $180 / 110 \mathrm{mmHg}$, if they had a history of cardiovascular disease or chronic kidney disease, if they had a serum electrolyte abnormality within the prior 6 months, if they had a known intolerance or drug allergy to the $\mathrm{BP}$ classes being tested, if they were prescribed BP medications for an indication other than hypertension, if they were non-English speaking, if they did not have daily access to email or text messaging, if they had physical or cognitive impairments that precluded them from self-monitoring BP or side effects, or if they were unavailable for follow-up. The Institutional Review Board of Columbia University Irving Medical Center approved the protocol, and patients provided written informed consent. The trial was registered on clinicaltrials.gov (NCT02744456).

\section{Procedures}

The baseline visit consisted of assessing demographic characteristics, medical history, and preferences for shared decisionmaking, ${ }^{16}$ as well as for having office BP measured according to a standardized research protocol. ${ }^{13}$ In the spirit of therapeutic precision, consented patients who were not already prescribed BP medications had the option of conducting 24-h ambulatory BP monitoring to exclude white coat hypertension before beginning the personalized trial. ${ }^{17}$ Eligible patients then met with the study clinician, a board-certified general internist (I.K.), to finalize the treatment sequence. Once selected, patients filled the BP medications for the trial from their usual pharmacies. Patients subsequently received daily text messages or emails, as per preference, that reminded them about the medication they were supposed to be taking and about self-assessments. At the conclusion of the personalized trial, patients met with the study clinician in person or by telephone to review visualizations of their self-monitored BP and side effect data and to state their treatment preferences. Data visualizations and treatment preference were also shared with the patient's treating clinician. At least 6 weeks later, patients were contacted by telephone to inquire about their current BP treatment regimens.

\section{Outcomes}

Blood Pressure. BP was measured by patients twice in the morning and twice at night during the second week of each 2week treatment period using a valid home BP device (Omron model BP1711T, Omron Healthcare, Inc.). ${ }^{18}$ This protocol allowed 1 week for washout of prior medication and 1 week for the onset of the next medication prior to assessments. Patients received instruction in how to self-measure BP during enrollment. Of note, home BP devices also recorded pulse, and one participant with symptoms of palpitations was provided with summary data on pulse, as per her request.

Side Effects. During the second of each 2-week treatment period, patients received an end-of-the-day email or text message asking if they had experienced side effects from their BP medication. If yes, patients were then asked to rate how much the side effects interfered with their physical (e.g., headaches, dizziness, leg swelling), cognitive (e.g., tiredness, ability to think clearly), and emotional (e.g., feeling nervous or upset) health, respectively, using a 5-item Likert scale (1 ["not at all"] to 5 ["extremely"]). 
Compliance. Compliance was assessed by counting the number of BP readings and side effect assessments completed by patients. Patients were categorized as compliant if they provided a minimum of $12 \mathrm{BP}$ readings and 4 side effect assessments for each 1-week assessment period.

Perceived helpfulness. After reviewing their results with the study clinician, patients were asked to rate the helpfulness of participating in the personalized trial with respect to their BP (response options: 1 ["not at all helpful"] to 5 ["extremely helpful"]) and whether they would recommend a personalized trial of BP medications to other hypertensive patients (response options: yes, no, maybe).

\section{Statistical Analysis and Sample Size}

Frequency and severity of side effects were compared visually using column graphs, without tests of statistical significance. Means and standard deviations of BP for each BP medication treatment period were similarly visualized using a column graph. The statistical significance of differences in effects of medication on SBP were assessed post hoc using generalized estimating equations (GEEs) with an unstructured variancecovariance matrix for measures on the same day. This model accounted for the possible autocorrelation between BP readings 1 day apart and for the time of day (a.m. versus p.m.) as covariates.

We enrolled ten adults to have a sufficient number of participants to assess the feasibility of conducting personalized trials of BP medications. For the personalized trials' methodology, each trial represents a single experiment, and sample size refers to the number of outcome measurements per treatment period and the number of treatment repetitions in a given trial. The number of BP readings and treatment repetitions planned for these personalized trials was based on expert recommendations for home BP monitoring protocols, ${ }^{19}$ an understanding of the amount of time needed for the onset and washout of the prototypical BP medications based on each medication's half-life, and estimations about the maximum tolerated duration of the trial to maintain patient engagement.

\section{RESULTS}

\section{Participant Flow and Baseline Characteristics}

Of 13 patients screened for participation, 10 provided informed consent and 3 declined to participate after learning about the study (Online Appendix 1, Supplemental Figure 1). One was withdrawn after being diagnosed with white coat hypertension via ambulatory BP monitoring, and two dropped out before initiating the trial. Of the seven patients who initiated, the mean age was 58 years, and five of seven were women (Table 1). All patients had at least a high school diploma. Participants had a median of two chronic medical
Table 1 Characteristics of Patients Who Consented and Completed the $N$-of-1 Trial of Blood Pressure Medications

\begin{tabular}{|c|c|c|}
\hline Characteristic & $\begin{array}{l}\text { All } \\
\text { consented } \\
\text { patients } \\
(N=10)\end{array}$ & $\begin{array}{l}\text { All patients } \\
\text { who } \\
\text { completed the } \\
\text { personalized } \\
\text { trial } \\
(N=7)\end{array}$ \\
\hline Age in years, mean (SD) & $56.9(10.9)$ & $57.7(11.4)$ \\
\hline Female & $7(70.0)$ & $5(71.4)$ \\
\hline Hispanic & $1(10.0)$ & $1(14.3)$ \\
\hline Non-white & $5(50.0)$ & $4(57.1)$ \\
\hline \multicolumn{3}{|l|}{ Education } \\
\hline Less than high school & $0(0)$ & $0(0)$ \\
\hline High school & $3(30.0)$ & $2(28.6)$ \\
\hline College diploma & $7(70.0)$ & $5(71.4)$ \\
\hline \multicolumn{3}{|l|}{ Employment } \\
\hline Working & $7(70.0)$ & $4(57.1)$ \\
\hline Retired & $3(30.0)$ & $3(42.9)$ \\
\hline \multicolumn{3}{|l|}{ Health insurance type } \\
\hline Commercial & $6(70.0)$ & $4(57.1)$ \\
\hline Medicare & $3(30.0)$ & $3(42.9)$ \\
\hline Medicaid & $1(10.0)$ & $0(0)$ \\
\hline $\begin{array}{l}\text { Number of chronic medical } \\
\text { conditions, median (range) }\end{array}$ & $2(1-4)$ & $2(1-4)$ \\
\hline Total medications, median (range) & $2(0-3)$ & $2(0-3)$ \\
\hline \multicolumn{3}{|l|}{ Number of BP medications } \\
\hline None & $3(30.0)$ & $1(14.3)$ \\
\hline One & $7(70.0)$ & $6(85.7)$ \\
\hline $\begin{array}{l}\text { Systolic blood pressure at baseline } \\
\text { in mmHg, mean (SD) }\end{array}$ & $135.1(11.6)$ & $130.1(9.8)$ \\
\hline $\begin{array}{l}\text { Diastolic blood pressure at baseline } \\
\text { in } \mathrm{mmHg} \text {, mean (SD) }\end{array}$ & $86(7.0)$ & $85.1(6.9)$ \\
\hline \multicolumn{3}{|l|}{ Shared decision-making preference } \\
\hline Doctor decides & $0(0)$ & $0(0)$ \\
\hline Doctor considers my ideas & $1(10.0)$ & $1(14.3)$ \\
\hline Decide together with doctor & $8(80.0)$ & 5 (71.4) \\
\hline I decide & $1(10.0)$ & $1(14.3)$ \\
\hline
\end{tabular}

Data are presented as $N(\%)$ unless otherwise specified

conditions. Most patients (six of seven) were prescribed one $\mathrm{BP}$ medication prior to the start of the trial, and the mean office $\mathrm{BP}$ at enrollment was $130 / 85 \mathrm{mmHg}$. The majority of participants (five of seven) preferred shared decision-making with their personal clinicians.

\section{Outcomes of Personalized Trials of BP Medications}

The treatment sequence and outcomes of each personalized trial are shown in Table 2. Each patient's daily and mean systolic BP readings over the course of the personalized trial are shown in Fig. 1. We next describe the results of each patient's personalized trial, as each trial can be viewed as its own experiment.

Patient A, who was prescribed HCTZ-bisoprolol prior to the trial, compared this medication with amlodipine, losartan, and metoprolol (Online Appendix 2, Supplemental Figure 2a). This patient learned that HCTZbisoprolol and amlodipine better lowered her BP than losartan and metoprolol. She additionally used pulse data to learn that the HCTZ-bisoprolol best reduced her pulse and associated palpitations. This patient ultimately preferred HCTZ-bisoprolol and found the trial extremely helpful. 
Table 2 Characteristics and Outcomes of Personalized Trials of Blood Pressure Medications

\begin{tabular}{|c|c|c|c|c|c|c|c|}
\hline 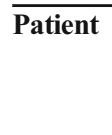 & $\begin{array}{l}\text { Treatment } \\
\text { prior to trial }\end{array}$ & $\begin{array}{l}\text { Treatment } \\
\text { sequence }\end{array}$ & $\begin{array}{l}\text { Percent of days } \\
\text { monitored, with } \\
\text { side effects }\end{array}$ & $\begin{array}{l}\text { Treatment } \\
\text { preference at } \\
\text { the } \\
\text { end of trial }\end{array}$ & $\begin{array}{l}\text { Prescribed }>6 \\
\text { weeks after } \\
\text { trial }\end{array}$ & $\begin{array}{l}\text { Helpfulness of } \\
\text { participation }\end{array}$ & $\begin{array}{l}\text { Recommend } \\
\text { trial to others }\end{array}$ \\
\hline A & $\begin{array}{l}\text { HCTZ- } \\
\text { bisoprolol }\end{array}$ & $\begin{array}{l}\text { HB-A1-Lo-Lo- } \\
\text { A2-HB-M }\end{array}$ & $\begin{array}{l}\mathrm{HB}=0 \%(0 \text { of } \\
14) \\
\mathrm{A} 1=0 \%(0 \text { of } 3) \\
\mathrm{Lo}=0 \%(0 \text { of } 14) \\
\mathrm{A} 2=0 \%(0 \text { of } 7) \\
\mathrm{M}=0 \%(0 \text { of } 1)\end{array}$ & HCTZ-bisoprolol & $\begin{array}{l}\text { HCTZ- } \\
\text { bisoprolol }\end{array}$ & $\begin{array}{l}\text { Extremely } \\
\text { helpful }\end{array}$ & Yes \\
\hline B & Lisinopril & Lo-A-H & $\begin{array}{l}\text { Lo }=0 \%(0 \text { of } 7) \\
A=29 \%(2 \text { of } 7) \\
H=20 \%(1 \text { of } 5)\end{array}$ & No preference & Lisinopril & $\begin{array}{l}\text { Somewhat } \\
\text { helpful }\end{array}$ & Yes \\
\hline $\mathrm{C}$ & Amlodipine & Lo-A-H & $\begin{array}{l}\mathrm{Lo}=25 \%(1 \text { of } 4) \\
\mathrm{A}=\mathrm{n} / \mathrm{a} * \\
\mathrm{H}=\mathrm{n} / \mathrm{a}\end{array}$ & Losartan & Losartan & Very helpful & Yes \\
\hline $\mathrm{D}$ & None & $\begin{array}{l}\text { Lo-A-H-H-A- } \\
\text { Lo-A-Lo-H }\end{array}$ & $\begin{array}{l}\mathrm{Lo}=0 \%(0 \text { of } 17) \\
\mathrm{A}=0 \%(0 \text { of } 19) \\
\mathrm{H}=0 \%(0 \text { of } 17)\end{array}$ & HCTZ & $\mathrm{HCTZ}$ & $\begin{array}{l}\text { Extremely } \\
\text { helpful }\end{array}$ & Yes \\
\hline E & Lisinopril & Lo-Li-H & $\begin{array}{l}\mathrm{Lo}=0 \%(0 \text { of } 6) \\
\mathrm{Li}=0 \%(0 \text { of } 4) \\
\mathrm{H}=0 \%(0 \text { of } 7)\end{array}$ & Lisinopril & Lisinopril & Not at all helpful & Yes \\
\hline $\mathrm{F}$ & $\begin{array}{l}\text { HCTZ- } \\
\text { triamterene }\end{array}$ & $\begin{array}{l}\text { HT-Lo-A-A-Lo- } \\
\text { HT }\end{array}$ & $\begin{array}{l}\mathrm{HT}=0 \%(0 \text { of } \\
13) \\
\mathrm{Lo}=0 \%(0 \text { of } 11) \\
\mathrm{A}=9 \%(1 \text { of } 11)\end{array}$ & $\begin{array}{l}\text { HCTZ- } \\
\text { triamterene }\end{array}$ & $\begin{array}{l}\text { HCTZ- } \\
\text { triamterene }\end{array}$ & Very helpful & Yes \\
\hline G & Chlorthalidone & $\mathrm{C}-\mathrm{H}$ & $\begin{array}{l}\mathrm{C}=0 \%(0 \text { of } 6) \\
\mathrm{H}=0 \%(0 \text { of } 7)\end{array}$ & Chlorthalidone & Chlorthalidone & $\begin{array}{l}\text { Extremely } \\
\text { helpful }\end{array}$ & Yes \\
\hline
\end{tabular}

A amlodipine, A1 low-dose amlodipine, A2 medium-dose amlodipine, C chlorthalidone, H hydrochlorothiazide, HB hydrochlorothiazide-bisoprolol, HT hydrochlorothiazide-triamterene, Lo losartan, Li Lisinopril, M metoprolol, n/a data not provided, SBP systolic blood pressure

*Participant self-reported prominent leg edema while taking amlodipine but did not complete self-assessments electronically

Patient B, who was prescribed lisinopril before the trial, compared three different medications (losartan, amlodipine, and HCTZ; Online Appendix 2, Supplemental Figure 2b). This patient learned that losartan, amlodipine, and HCTZ similarly lowered her BP and that losartan and HCTZ were associated with mild physical side effects. He had no preference for any of the trialed medications, found the trial somewhat helpful, and ultimately remained on lisinopril after the trial. Patient $\mathrm{C}$, who was prescribed amlodipine before the trial, compared three medications (losartan, amlodipine, and HCTZ). This patient found that losartan and amlodipine both lowered her BP better than HCTZ, but that only amlodipine caused leg edema. This patient preferred losartan after the trial and found the trial very helpful. Patient D, who was not prescribed a BP medication before the trial, compared three medications (losartan, amlodipine, and HCTZ). This patient learned that HCTZ best lowered her BP and had no side effects. This patient found the trial extremely helpful. Patient E, who was taking lisinopril prior to the trial, compared three medications (losartan, lisinopril, and HCTZ). This patient learned that both lisinopril and losartan better lowered her BP than HCTZ, and neither lisinopril nor losartan had side effects. This patient preferred lisinopril after the trial and concluded that, even though the trial was unhelpful for managing her $\mathrm{BP}$, she still would recommend such trials to others. Patient $\mathrm{F}$, who was taking HCTZ-triamterene prior to the trial, compared three medications (HCTZ-triamterene, amlodipine, and losartan). This patient learned that HCTZ- triamterene best lowered her BP and that all of the medications were well tolerated. This patient preferred HCTZtriamterene at the end of the trial and concluded that the trial was very helpful. Patient $G$, who was prescribed chlorthalidone prior to the trial, compared chlorthalidone with HCTZ as per his preference. This patient learned that the two diuretics similarly lowered his BP and neither produced side effects. This patient concluded that the trial was extremely helpful and should be recommended to others.

Pooling results across the series of personalized trials, all patients complied with home BP monitoring, and the majority (six of seven) complied with side effect tracking. Of six patients prescribed a BP medication before the personalized trial, one preferred a different medication at the end of a trial. Nearly all patients (six of seven) found participation in the trials at least somewhat helpful to their BP, and all patients said they would recommend such trials to other hypertensive patients. At least 6 weeks after the trial, all patients were prescribed a BP medication concordant with their end-of-trial preferences.

The estimation of BP lowering effects of medications was similar whether BP readings were averaged, a more pragmatic approach, or were modeled using sophisticated time series analyses that may be more cumbersome for clinicians to implement (Online Appendix 2, Supplemental Table 1). Statistically significant differences in BP between at least one pair of BP medications were found in four of the seven personalized trials. 

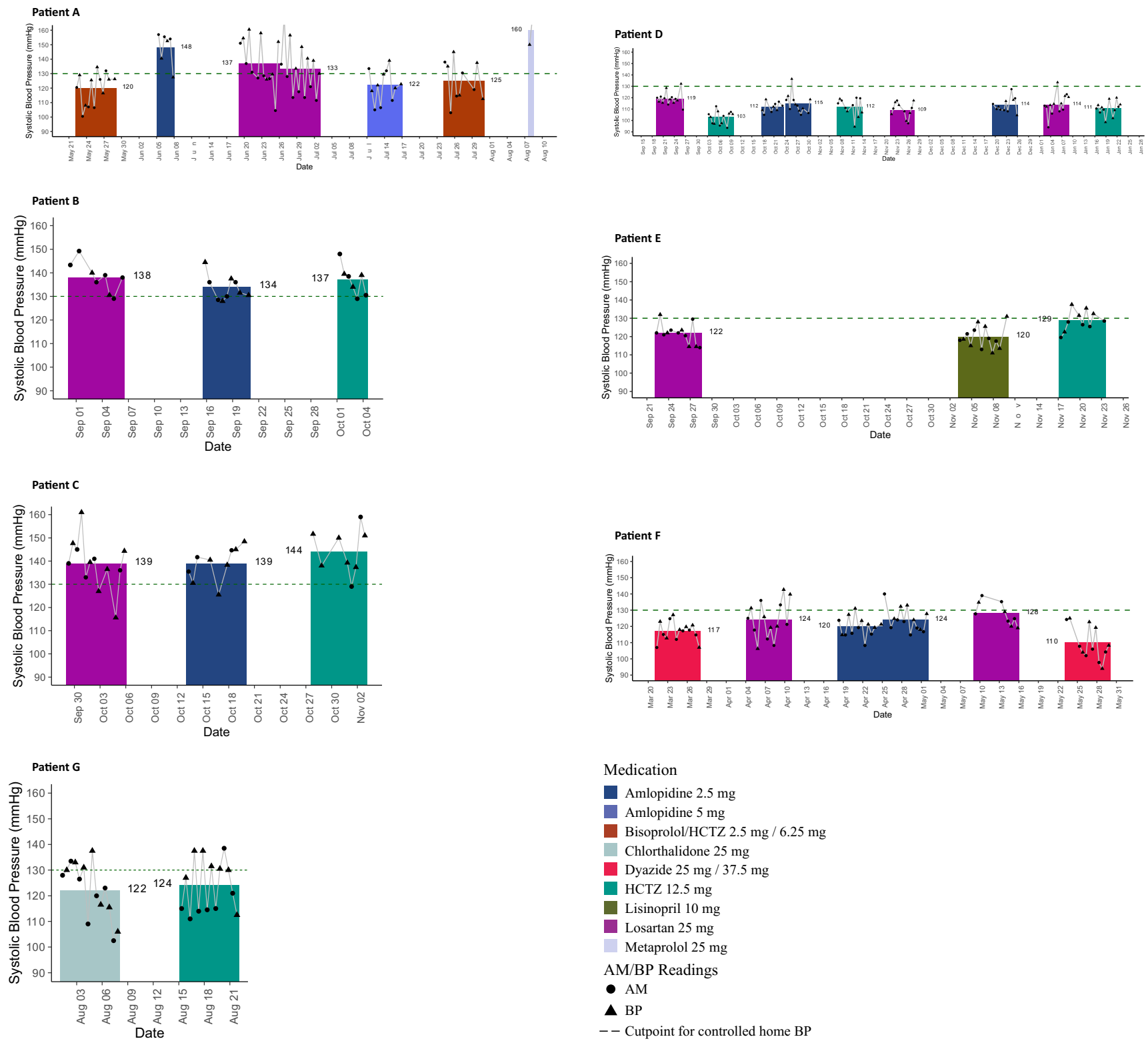

Fig. 1 Morning/night and overall mean systolic blood pressure during each personalized trial. The black circles and triangles show the average of morning (a.m.) and night (p.m.) systolic blood pressure (BP) readings, respectively, that were self-measured using a home BP device. These readings came from the second week of taking each medication. The gaps between the columns reflect the time period during which BP was not measured. This gap allowed for the time on the prior medication to wash out and the time for the new medication to have an onset of action. The columns reflect the mean of all systolic BP readings taken during that time period for each medication. The dashed line refers to goal BP according to the 2017 American College of Cardiology/American Heart Association high BP guidelines.

\section{DISCUSSION}

In this pilot study, we gained preliminary evidence for the feasibility and benefit of using personalized trials to select BP medications. Nearly all patients who participated in the trials were compliant with the protocol. Further, nearly all patients found the trials to be helpful, and all agreed they would recommend such trials to other hypertensive patients. Importantly, all patients were prescribed their preferred BP medication after the personalized trial, suggesting that the data obtained through personalized trials could influence prescribing decisions by treating providers. Finally, the series of personalized trials revealed that preferences for BP medications differed between patients. Three patients preferred thiazide diuretics, two patients preferred losartan, one patient preferred amlodipine, and one patient preferred a combination of drugs (bisoprolol-hydrochlorothiazide). Thus, we concluded that such trials may truly help personalize treatment selection such that patients end up on more satisfying BP medications than if medications were selected according to the usual clinical approach.

To our knowledge, there are few published reports of personalized trials of BP treatments. Estrada and Young ${ }^{20}$ presented the results of a personalized trial in which one patient compared the effect of garlic and placebo on their BP. Others 
have used crossover-design experiments to study the heterogeneity of treatment effects for BP medications. ${ }^{19,21}$ These trials, however, did not evaluate the influence of participation in such studies on patient preferences for BP medications.

There were several features of our personalized trial design that could facilitate uptake by clinicians. First, BP medications were not blinded. Masking treatments can be expensive and burdensome to implement, and patients hold unfavorable attitudes toward blinding. ${ }^{22-24}$ Second, this trial did not involve randomization which can be challenging to implement without the assistance of specialized software. Instead, a balanced treatment sequence was used to protect against bias from time effects. Third, the tools used to collect data-home BP monitors and text messages or emails - are readily available to most patients and clinicians. In the future, the development of smartphone applications that guide patients through BP medication treatment sequences, structure the collection of BP and side effect data, and automate data visualizations and analyses could enhance the scalability of such approaches. ${ }^{25}$

While this pilot study suggested that personalized trials of BP medications are promising, there were also limitations of our findings. Feasibility was assessed on a small number of patients with few chronic medical conditions, limiting generalizability. Future evaluations of personalized trials of BP medications should seek to enroll larger samples of diverse patients. The feasibility of implementing such trials into practice from a clinician and health system perspective was not assessed. Similarly, the study did not assess the impact of personalized trials on long-term adherence to treatment, BP control, or health care costs. The personalized trials tested in this study did not require treatment repetitions, randomization, or blinding which may have influenced the robustness of findings. Only one of the six patients that were prescribed a BP medication prior to the personalized trial preferred a different BP medication after the trial. Nevertheless, all but one of these patients found the trial at least somewhat helpful, and one patient who found the trial unhelpful still recommended the trial to others. It may be that such participants still benefited by gaining a greater understanding of the benefits and harms of BP medications. Future personalized trials of BP medications should include more detailed assessments of the impact of participation on attitudes toward and adherence to BP medications. Future studies should also incorporate direct comparisons of the personalized trial approach with usual hypertension care.

In conclusion, this proof-of-concept pilot study of pragmatic personalized trials of BP medications suggested that this patient-centered approach is feasible and helpful to most patients, although this approach needs to be tested in larger samples of patients to investigate its usefulness more thoroughly. The pragmatic design may provide a road map for increasing the uptake of personalized trials. Overall, our study suggests that personalized trials hold promise for changing the way BP medications are selected in clinical practice.
Corresponding Author: Ian M. Kronish, MD, MPH; Center for Behavioral Cardiovascular Health, Columbia University Irving Medical Center, 622 West 168th Street, PH9-311, New York, NY 10032, USA (e-mail: ik2293@columbia.edu).

Funding The study was funded by the National Center for Advancing Translational Sciences (U01 TROO1873). Dr. Kronish and Dr. Davidson received additional support from the National Library of Medicine (RO1 LMO12836).

\section{Compliance with Ethical Standards:}

Conflict of Interest: The authors declare that they do not have a conflict of interest.

Publisher's Note: Springer Nature remains neutral with regard to jurisdictional claims in published maps and institutional affiliations.

\section{REFERENCES}

1. Tarn DM, Heritage J, Paterniti DA, Hays RD, Kravitz RL, Wenger NS. Physician communication when prescribing new medications. Arch Intern Med. 2006;166(17):1855-62.

2. Gu Q, Burt VL, Dillon CF, Yoon S. Trends in antihypertensive medication use and blood pressure control among United States adults with hypertension: the National Health And Nutrition Examination Survey, 2001 to 2010. Circulation. 2012;126(17):2105-14.

3. Dickerson JE, Hingorani AD, Ashby MJ, Palmer CR, Brown MJ. Optimisation of antihypertensive treatment by crossover rotation of four major classes. Lancet. 1999;353(9169):2008-13.

4. Deary AJ, Schumann AL, Murfet H, Haydock SF, Foo RS, Brown MJ. Double-blind, placebo-controlled crossover comparison of five classes of antihypertensive drugs. J Hypertens. 2002;20(4):771-7.

5. Edmonds D, Huss R, Jeck T, Mengden T, Schubert M, Vetter W. Individualizing antihypertensive therapy with enalapril versus atenolol: the Zurich experience. J Hypertens Suppl. 1990;8(4):S49-52.

6. Waeber B, Burnier M, Nussberger J, Brunner HR. Trials using a crossover design and ambulatory blood pressure recordings to determine the efficacy of antihypertensive agents in individual patients. J Hypertens Suppl. 1990;8(4):S37-41

7. Gong Y, MCDonough CW, Wang Z, Hou W, Cooper-DeHoff RM, Langaee TY, et al. Hypertension susceptibility loci and blood pressure response to antihypertensives: results from the pharmacogenomic evaluation of antihypertensive responses study. Circ Cardiovasc Genet. 2012;5(6):686-91.

8. Cooper-DeHoff RM, Johnson JA. Hypertension pharmacogenomics: in search of personalized treatment approaches. Nat Rev Nephrol. 2016;12(2): 110-22

9. Duan N, Kravitz RL, Schmid CH. Single-patient (n-of-1) trials: a pragmatic clinical decision methodology for patient-centered comparative effectiveness research. J Clin Epidemiol. 2013;66(8 Suppl):S21-8.

10. Kravitz RL DN, eds, and the DEcIDE Methods Center N-of-1 Guidance Panel (Duan N, Eslick I, Gabler NB, Kaplan HC, Kravitz RL, Larson EB, Pace WD, Schmid CH, Sim I, Vohra S). Design and Implementation of Nof-1 Trials: A User's Guide. AHRQ Publication No.13(14)-EHC122-EF. Rockville: MD; February 2014.

11. Guyatt GH, Haynes RB, Jaeschke RZ, Cook DJ, Green L, Naylor CD, et al. Users' Guides to the Medical Literature: XXV. Evidence-based medicine: principles for applying the Users' Guides to patient care. Evidence-Based Medicine Working Group. JAMA. 2000;284(10):1290-6.

12. Lillie EO, Patay B, Diamant J, Issell B, Topol EJ, Schork NJ. The n-of1 clinical trial: the ultimate strategy for individualizing medicine? Per Med. 2011;8(2):161-73.

13. Shimbo D, Abdalla M, Falzon L, Townsend RR, Muntner P. Role of Ambulatory and Home Blood Pressure Monitoring in Clinical Practice: A Narrative Review. Ann Intern Med. 2015;163(9):691-700.

14. Vohra S, Shamseer L, Sampson M, Bukutu C, Schmid $\mathbf{C H}$, Tate $\mathbf{R}$, et al. CONSORT extension for reporting N-of-1 trials (CENT) 2015 Statement. J Clin Epidemiol. 2016;76:9-17. 
15. Kravitz RL DN, Vohra S, Li J, the DEcIDE Methods Center N-of-1 Guidance Panel. Introduction to N-of-1 Trials: Indications and Barriers. In: Kravitz RL DN, eds, and the DEcIDE Methods Center N-of-1 Guidance Panel (Duan N, Eslick I, Gabler NB, Kaplan HC, Kravitz RL, Larson EB, Pace WD, Schmid CH, Sim I, Vohra S), ed. Design and Implementation of N-of-1 Trials: A User's Guide. AHRQ Publication No. 13(14)-EHC122-EF Rockville, MD: Agency foe Healthcare Research and Quality; 2014:1-11.

16. Degner LF, Sloan JA, Venkatesh P. The Control Preferences Scale. Can J Nurs Res. 1997;29(3):21-43.

17. Siu AL, Force USPST. Screening for high blood pressure in adults: U.S. Preventive Services Task Force recommendation statement. Ann Intern Med. 2015; 163(10):778-86.

18. Pickering TG, Miller NH, Ogedegbe G, Krakoff LR, Artinian NT, Goff D, et al. Call to action on use and reimbursement for home blood pressure monitoring: executive summary: a joint scientific statement from the American Heart Association, American Society Of Hypertension, and Preventive Cardiovascular Nurses Association. Hypertension. 2008;52(1):1-9

19. Chatellier G, Day M, Bobrie G, Menard J. Feasibility study of N-of-1 trials with blood pressure self-monitoring in hypertension. Hypertension. 1995;25(2):294-301.

20. Estrada CA, Young MJ. Patient preferences for novel therapy: an N-of-1 trial of garlic in the treatment for hypertension. J Gen Intern Med. 1993;8(11):619-21.
21. Materson BJ, Reda DJ, Preston RA, Cushman WC, Massie BM, Freis ED, et al. Response to a second single antihypertensive agent used as monotherapy for hypertension after failure of the initial drug. Department of Veterans Affairs Cooperative Study Group on Antihypertensive Agents. Arch Intern Med. 1995;155(16): 1757-62.

22. Kronish IM, Alcántara C, Duer-Hefele J, St Onge T, Davidson KW, Carter EJ, et al. Patients and primary care providers identify opportunities for personalized (N-of-1) trials in the mobile health era. J Clin Epidemiol. 2017;89:236-237.

23. Kravitz RL, Duan N, Niedzinski EJ, Hay MC, Subramanian SK, Weisner TS. What ever happened to $\mathrm{N}-\mathrm{of}-1$ trials? Insiders' perspectives and a look to the future. Milbank Q. 2008;86(4):533-55.

24. Kravitz RL, Paterniti DA, Hay MC, Subramanian S, Dean DE, Weisner T, et al. Marketing therapeutic precision: Potential facilitators and barriers to adoption of n-of-1 trials. Contemp Clin Trials. 2009;30(5):436-45.

25. Barr C, Marois M, Sim I, Schmid CH, Wilsey B, Ward D, et al. The PREEMPT study - evaluating smartphone-assisted n-of- 1 trials in patients with chronic pain: study protocol for a randomized controlled trial. Trials. 2015;16:67. 